JOURNAL OF THE

AMERICAN MATHEMATICAL SOCIETY

Volume 14, Number 2, Pages 263-278

S 0894-0347(00)00364-7

Article electronically published on December 21, 2000

\title{
AN EULERIAN-LAGRANGIAN APPROACH FOR INCOMPRESSIBLE FLUIDS: LOCAL THEORY
}

\author{
PETER CONSTANTIN
}

\section{INTRODUCTION}

The three dimensional Euler equations are evolution equations for the three velocity components $u(x, t)$,

$$
\frac{\partial u}{\partial t}+u \cdot \nabla u+\nabla p=0,
$$

coupled with a fourth equation, $\nabla \cdot u=0$, expressing incompressibility. In this Eulerian formulation the velocity $u$ and pressure $p$ are recorded at fixed locations $x \in \mathbf{R}^{3}$. The velocities and pressure vanish at infinity or are periodic. The pressure is determined using incompressibility. The equation is conservative and the total kinetic energy, $\int|u|^{2} d x$, is a constant of motion.

The Euler equations can be studied in terms of the vorticity ([1). The vorticity is a vector $\omega=\nabla \times u$ corresponding to the anti-symmetric part of the gradient matrix $\nabla u$. It obeys a quadratic equation, whose nature is such that the magnitude of the vorticity may increase in time. If the amplification is not rapid enough, then a well-known criterion ([2]) guarantees that no blow up can occur: if

$$
\int_{0}^{T} \sup _{x}|\omega(x, t)| d t<\infty
$$

and the initial data are smooth, then the solution is smooth on the time interval $[0, T]$. The vorticity equation can be interpreted as the vanishing of a commutator

$$
\left[D_{t}, \Omega\right]=0
$$

where

$$
D_{t}=\frac{\partial}{\partial t}+u \cdot \nabla
$$

is the material derivative and

$$
\Omega=\omega \cdot \nabla .
$$

The characteristics of the first order differential operator $\Omega$ are vortex lines; the characteristics of the material derivative $D_{t}$ are Lagrangian particle paths. The Lagrangian variables are the path maps $a \mapsto X(a, t)$. The connection between the Lagrangian description and the Eulerian one is given by the relations

$$
u(x, t)=\frac{\partial X(a, t)}{\partial t}, x=X(a, t) .
$$

Received by the editors September 27, 1999.

2000 Mathematics Subject Classification. Primary 76B03, 37K65, 35Q30, 35L65.

Key words and phrases. Euler equations, blow up. 
In this paper we discuss a description of the Euler equations as a system of three coupled active vector equations. The description concerns Lagrangian quantities computed in Eulerian variables and thus combines the physical significance of the Lagrangian description with the analytical advantages of the Eulerian description. The description bears similarities to the Clebsch variable representation. The Clebsch variables are a pair of scalars, $\theta, \varphi$, that are constant on particle paths and can be used to reconstruct the velocity via

$$
u^{i}(x, t)=\theta(x, t) \frac{\partial \varphi(x, t)}{\partial x_{i}}-\frac{\partial n(x, t)}{\partial x_{i}} .
$$

This interesting representation is somewhat restrictive: not all solutions can be represented in this manner. That is because the Clebsch variables impose special constraints on helicity. Helicity is the scalar product of velocity and vorticity $h=$ $u \cdot \omega$. Although $h$ itself is not conserved on particle paths, the integrals

$$
\int_{T} h(x, t) d x=c
$$

are constants of motion, for any vortex tube $T$. A vortex tube $T$ is a time evolving region in space (not necessarily simply connected) whose boundary is at each point parallel to the vorticity, $\omega \cdot \nu=0$ where $\nu$ is the normal to $\partial T$ at $x \in \partial T$. The constants $c$ reflect the degree of topological complexity of the flow ([3]) and in general are nontrivial but they vanish identically for flows that admit a Clebsch variables representation. Indeed, for such flows the helicity is the divergence of a field that is parallel to the vorticity $h=-\nabla \cdot(n \omega)$. Topological properties of streamlines and vortex tubes are relevant to hydrodynamic stability ([4]) and turbulence ([5], [6]). The description of the flow that allows for arbitrary vortex structures is based on formula (15) (7], 8], 9], 10]) that was used for numerical computations. Somewhat related Hamiltonian formulations have been introduced by several authors ([11], [12], [13], [14]).

\section{EULERIAN-LAGRANGIAN DESCRIPTION}

The Lagrangian formulation of the Euler equations describes the flow in terms of a volume preserving diffeomorphism, the map $a \mapsto X(a, t)$. The curve $t \mapsto X(a, t)$ is the Lagrangian path at label $a$ and obeys Newton's law

$$
\frac{\partial^{2} X(a, t)}{\partial t^{2}}=F_{X}(a, t)
$$

The incompressibility condition for the map is

$$
\operatorname{det}\left(\nabla_{a} X\right)=1 .
$$

The initial condition sets the labels at the initial time:

$$
X(a, 0)=a .
$$

The forces $F_{X}$ in (3) are

$$
F_{X}(a, t)=-\left(\nabla_{x} p\right)(X(a, t))=-\left[\left(\nabla_{a} X(a, t)\right)^{*}\right]^{-1}\left(\nabla_{a} \tilde{p}\right)(a, t)
$$

with $\tilde{p}(a, t)=p(X(a, t))$ and where $p$ is the Eulerian pressure. The notation $M^{*}$ means the transpose of the matrix $M,\left(M^{*}\right)^{-1}$ its inverse. Multiplying (3) by 
$\left(\nabla_{a} X\right)^{*}$ we obtain

$$
\frac{\partial^{2} X(a, t)}{\partial t^{2}}\left(\nabla_{a} X(a, t)\right)^{*}=-\left(\nabla_{a} \tilde{p}\right)(a, t)
$$

or, on components

$$
\frac{\partial^{2} X^{j}(a, t)}{\partial t^{2}} \frac{\partial X^{j}(a, t)}{\partial a_{i}}=-\frac{\partial \tilde{p}(a, t)}{\partial a_{i}} .
$$

Pulling out a time derivative in the left-hand side we obtain

$$
\frac{\partial}{\partial t}\left[\frac{\partial X^{j}(a, t)}{\partial t} \frac{\partial X^{j}(a, t)}{\partial a_{i}}\right]=-\frac{\partial \tilde{q}(a, t)}{\partial a_{i}}
$$

where

$$
\tilde{q}(a, t)=\tilde{p}(a, t)-\frac{1}{2}\left|\frac{\partial X(a, t)}{\partial t}\right|^{2} .
$$

We integrate (8) in time, fixing the label $a$ :

$$
\frac{\partial X^{j}(a, t)}{\partial t} \frac{\partial X^{j}(a, t)}{\partial a_{i}}=u_{(0)}^{i}(a)-\frac{\partial \tilde{n}(a, t)}{\partial a_{i}}
$$

where

$$
\tilde{n}(a, t)=\int_{0}^{t} \tilde{q}(a, s) d s
$$

and

$$
u_{(0)}(a)=\frac{\partial X(a, 0)}{\partial t}
$$

is the initial velocity. Note that $\tilde{n}$ has dimensions of circulation or of kinematic viscosity (length squared per time). The conservation of circulation

$$
\oint_{\gamma} \frac{\partial X(\gamma, t)}{\partial t} \cdot d \gamma=\oint_{\gamma} \frac{\partial X(\gamma, 0)}{\partial t} \cdot d \gamma
$$

follows directly from the form (10). Let us consider

$$
A(x, t)=X^{-1}(x, t),
$$

the "back-to-labels" map, and note that it forms a vector of active scalars (an active vector)

$$
D_{t} A=\frac{\partial A}{\partial t}+u \cdot \nabla A=0 .
$$

Turning to (101), multiplying by $\left[\left(\nabla_{a} X(a, t)\right)^{*}\right]^{-1}$ and reading at $a=A(x, t)$ we obtain the formula

$$
u^{i}(x, t)=\left(u_{(0)}^{j}(A(x, t))\right) \frac{\partial A^{j}(x, t)}{\partial x_{i}}-\frac{\partial n(x, t)}{\partial x_{i}}
$$

where

$$
n(x, t)=\tilde{n}(A(x, t)) .
$$


Equation (15) shows that the general Eulerian velocity can be written in a form that generalizes the Clebsch variable representation:

$$
u=(\nabla A)^{*} B-\nabla n
$$

where $B=u_{(0)}(A(x, t))$ is also an active vector,

$$
D_{t} B=0 \text {. }
$$

Conversely, and somewhat more generally, if one is given a pair of active vectors $A=$ $\left(A^{1}(x, t), \cdots, A^{M}(x, t)\right)$ and $B=\left(B^{1}(x, t), \cdots, B^{M}(x, t)\right)$ of arbitrary dimension $M$, such that the active vector equations (14) and (18) hold, and if $u$ is given by

$$
u(x, t)=\sum_{k=1}^{M} B^{k}(x, t) \nabla_{x} A^{k}(x, t)-\nabla_{x} n
$$

with some function $n$, then it follows that $u$ solves the Euler equations

$$
\frac{\partial u}{\partial t}+u \cdot \nabla u+\nabla \pi=0
$$

where

$$
\pi=D_{t} n+\frac{1}{2}|u|^{2} .
$$

Indeed, the only thing one needs is the kinematic commutation relation

$$
D_{t} \nabla_{x} f=\nabla_{x} D_{t} f-\left(\nabla_{x} u\right)^{*} \nabla_{x} f
$$

that holds for any scalar function $f$. The kinematic commutation relation (20) is a consequence of the chain rule, so it requires no assumption other than smoothness. Differentiating (19) and using the active vector equations (14), (18) it follows that

$$
\begin{aligned}
D_{t}(u) & =-\sum_{k=1}^{M}\left(\left(\nabla_{x} u\right)^{*} \nabla_{x} A^{k}\right) B^{k}-\nabla_{x}\left(D_{t} n\right)+\left(\nabla_{x} u\right)^{*} \nabla n \\
& =-\nabla_{x}\left(D_{t} n\right)-\left(\nabla_{x} u\right)^{*}\left[\sum_{k=1}^{M}\left(\nabla_{x} A^{k}\right) B^{k}-\nabla_{x} n\right] \\
& =-\nabla_{x}\left(D_{t} n\right)-\left(\nabla_{x} u\right)^{*} u=-\nabla_{x}(\pi) .
\end{aligned}
$$

\section{The ACTive Vector Formulation}

The previous calculations can be summarized as follows: A function $u(x, t)$ solves the incompressible Euler equations if and only if it can be represented in the form $u=u_{A}$ with

$$
u_{A}^{i}(x, t)=\phi^{m}(A(x, t)) \frac{\partial A^{m}(x, t)}{\partial x_{i}}-\frac{\partial n_{A}(x, t)}{\partial x_{i}}
$$

and

$$
\nabla \cdot u_{A}=0
$$

where $A(x, t)$ solves the active vector equation

$$
\left(\partial_{t}+u_{A} \cdot \nabla\right) A=0,
$$

with initial data

$$
A(x, 0)=x .
$$


The function $\phi$ represents the initial velocity and the function $n_{A}(x, t)$ is determined up to additive constants by the requirement of incompressibility, $\nabla \cdot u_{A}=0$ :

$$
\Delta n_{A}(x, t)=\frac{\partial}{\partial x_{i}}\left\{\phi^{m}(A(x, t)) \frac{\partial A^{m}(x, t)}{\partial x_{i}}\right\} .
$$

The periodic boundary conditions are

$$
A\left(x+L e_{j}, t\right)=A(x, t)+L e_{j}, \quad n_{A}\left(x+L e_{j}, t\right)=n_{A}(x, t)
$$

with $e_{j}$ the standard basis in $\mathbf{R}^{3}$. In this case

$$
\delta_{A}(x, t)=x-A(x, t),
$$

$n_{A}(x, t)$, and $u_{A}(x, t)$ are periodic functions in each spatial direction. One may consider also the case of decay at infinity, requiring that $\delta_{A}, u_{A}$, and $n_{A}$ vanish sufficiently fast at infinity. The equation of state (21), (22) can be written as

$$
u_{A}=\mathbf{P}\left\{\phi^{m}(A(\cdot, t)) \nabla A^{m}(\cdot, t)\right\}=\mathbf{P}\left\{(\nabla A)^{*} \phi(A)\right\}
$$

where

$$
\mathbf{P}=\mathbf{1}-\nabla \Delta^{-1} \nabla
$$

is the Leray-Hodge projector (with appropriate boundary conditions) on divergence free functions.

The Eulerian pressure is determined, up to additive constants, by

$$
p(x, t)=\frac{\partial n_{A}(x, t)}{\partial t}+u_{A}(x, t) \cdot \nabla n_{A}(x, t)+\frac{1}{2}\left|u_{A}(x, t)\right|^{2} .
$$

The Jacobian obeys

$$
\operatorname{det}(\nabla A(x, t))=1
$$

The vorticity

$$
\omega_{A}(x, t)=\nabla \times u_{A}
$$

satisfies the Helmholtz equation

$$
D_{t}^{A} \omega_{A}=\omega_{A} \cdot \nabla u_{A}
$$

and is given by the Cauchy formula

$$
\omega_{A}(x, t)=[\nabla A(x, t)]^{-1} \zeta(A(x, t))
$$

where $\zeta=\nabla \times \phi$ is the initial vorticity.

The advantage of an active vector formulation is that $A$ has conserved distribution, that is, for any function $\Phi$

$$
\int \Phi(A(x, t)) d x=\text { const; }
$$

in particular $\|A(\cdot, t)\|_{L_{l o c}^{\infty}(d x)}$ is constant in time. 


\section{LOCAL EXISTENCE}

The proof of local existence of solutions to the Euler equations in the active vector formulation is relatively simple and the result can be stated economically. There exist of course other well-known proofs of local existence results in Lagrangian ([15]) and Eulerian ([16]) coordinates.

Theorem 1. Let $\phi$ be a divergence free $C^{1, \mu}$ periodic vector-valued function of three variables. There exist a time interval $[0, T]$ and a unique $C\left([0, T] ; C^{1, \mu}\right)$ spatially periodic vector-valued function $\delta(x, t)$ such that

$$
A(x, t)=x+\delta(x, t)
$$

solves the active vector formulation of the Euler equations,

$$
\begin{gathered}
\frac{\partial A}{\partial t}+u \cdot \nabla A=0, \\
u=\mathbf{P}\left\{(\nabla A(x, t))^{*} \phi(A(x, t))\right\}
\end{gathered}
$$

with initial datum $A(x, 0)=x$.

The same result holds if one replaces periodic boundary conditions with decay at infinity. Differentiating the active vector equation (23) we obtain the equation obeyed by the gradients

$$
D_{t}^{A}\left(\frac{\partial A^{m}}{\partial x_{i}}\right)+\frac{\partial u_{A}^{j}}{\partial x_{i}} \frac{\partial A^{m}}{\partial x_{j}}=0 .
$$

It is useful to denote by

$$
\mathbf{P}_{j l}=\delta_{j l}-\partial_{j} \Delta^{-1} \partial_{l}
$$

the matrix elements of the Leray-Hodge operator. Differentiating in the representation (26) and using the fundamental property

$$
\mathbf{P}_{j l} \frac{\partial f}{\partial x_{l}}=0
$$

we obtain

$$
\frac{\partial u_{A}^{j}}{\partial x_{i}}=\mathbf{P}_{j l}\left(\operatorname{Det}\left[\zeta(A) ; \frac{\partial A}{\partial x_{i}} ; \frac{\partial A}{\partial x_{l}}\right]\right) .
$$

Recall that the function $\zeta$ is the curl of $\phi$. This relation shows that the gradient of velocity can be expressed without use of second order derivatives of $A$ and is the key to local existence: the equation (30) can be seen as a cubic quasi-local equation on characteristics. Let us make these ideas more precise. We will consider the periodic case first. We write $C^{j, \mu}, j=0,1$, to denote the Hölder spaces of real-valued functions that are defined for all $x \in \mathbf{R}^{3}$ and are periodic with period $L$ in each direction. We denote by $\|f\|_{0, \mu}$ the $C^{0, \mu}$ norm:

$$
\|f\|_{0, \mu}=\sup _{x}|f(x)|+\sup _{x \neq y}\left\{|f(x)-f(y)|\left(\frac{L}{|x-y|}\right)^{\mu}\right\}
$$

and by $\|f\|_{1, \mu}$ the $C^{1, \mu}$ norm:

$$
\|f\|_{1, \mu}=\|f\|_{0, \mu}+L\|\nabla f\|_{0, \mu}
$$

where the notation $|\cdots|$ refers to modulus, Euclidean norm, and Euclidean norm for matrices, as appropriate. 
We break the solution of the problem into two parts, the map $\delta \rightarrow u$ and the map $u \rightarrow \delta$. We denote the first one $W$ :

$$
W[\delta, \phi](x, t)=\mathbf{P}\left\{(\mathbf{I}+\nabla \delta(x, t))^{*} \phi(x+\delta(x, t))\right\} .
$$

This map is linear in $\phi$ but nonlinear in $\delta$.

Proposition 1. The map $W[\delta, \phi]$ maps

$$
W:\left(C^{1, \mu}\right)^{3} \times\left(C^{1, \mu}\right)^{3} \rightarrow\left(C^{1, \mu}\right)^{3}
$$

continuously. There exist constants $C$ depending on $\mu$ alone so that

$$
\|W[\delta, \phi]\|_{0, \mu} \leq C\|\phi\|_{0, \mu}\left\{1+\|\nabla \delta\|_{0, \mu}\right\}^{2}
$$

and

$$
\|\nabla W[\delta, \phi]\|_{0, \mu} \leq C\|\nabla \times \phi\|_{0, \mu}\left\{1+\|\nabla \delta\|_{0, \mu}\right\}^{3}
$$

hold for any $\delta \in\left(C^{1, \mu}\right)^{3}, \phi \in\left(C^{1, \mu}\right)^{3}$.

For the proof we note that $W$ is made up from a number of operations. The first operation is the composition $\phi(x) \mapsto \phi(x+\delta(x))$. For a fixed $\delta \in\left(C^{1, \mu}\right)^{3}$ the map $x \mapsto x+\delta$ is Lipschitz. Composition with a Lipschitz change of variables maps $C^{0, \mu}$ into itself continuously (we say that it is a continuous endomorphism). The joint continuity of $[\phi, \delta] \mapsto \phi(x+\delta)$ in $C^{1, \mu}$ follows naturally. The second operation is a sum of products of functions (a matrix applied to a vector). This is a continuous operation because the Hölder spaces $C^{j, \mu}, j=0,1$, we chose are Banach algebras. The third and last operation is the linear operator $\mathbf{P}$, which is bounded in Hölder spaces. We need to consider also derivatives of $W$. We use the formula (32) and note that the expression for the gradient is made of similar operations as above and apply the same kind of reasoning. This finishes the proof.

Time does not play any role in this proposition because the equation of state $(\delta, \phi) \mapsto W[\delta, \phi]$ is time independent. The second half of the procedure does depend on time. Let us denote by $\Theta$ the map that associates to two continuous paths $t \mapsto \delta(\cdot, t)$ and $t \mapsto \phi(\cdot, t)$ a new path $t \mapsto \theta$; the path $t \mapsto \theta=\Theta[\delta, \phi]$ is obtained by solving the partial differential equation

$$
\frac{\partial \theta}{\partial t}+u \cdot \nabla \theta+u=0
$$

where

$$
u=W[\delta(\cdot, t), \phi(\cdot, t)],
$$

periodic boundary conditions are imposed on $\theta$ and zero initial data

$$
\theta(x, 0)=0
$$

are required. The Euler equation only requires the use of a time independent $\phi$, but allowing time dependent $\phi$ is very useful: one can thus treat more equations, in particular the Navier-Stokes equation. Let us consider the space

$$
\mathcal{P}_{T}=C\left([0, T],\left(C^{1, \mu}\right)^{3}\right)
$$

of continuous $\left(C^{1, \mu}\right)^{3}$-valued paths defined on a time interval $[0, T]$, endowed with the natural norm

$$
\|\theta\|_{1, \mathcal{P}}=\sup _{t}\|\theta(\cdot, t)\|_{1, \mu}
$$


We will also consider the weaker norm

$$
\|\theta\|_{0, \mathcal{P}}=\sup _{t}\|\theta(\cdot, t)\|_{0, \mu}
$$

$\Theta$ is nonlinear in both arguments.

Proposition 2. The map $\Theta[\delta, \phi]$ maps

$$
\Theta: \mathcal{P}_{T} \times \mathcal{P}_{T} \rightarrow \mathcal{P}_{T}
$$

and is continuous when the topology of the source space $\mathcal{P}_{T} \times \mathcal{P}_{T}$ is the natural product $C^{1, \mu}$ topology and the topology of the target space $\mathcal{P}_{T}$ is the weaker $C^{0, \mu}$ topology. Moreover, there exists a constant $C$ depending on $\mu$ alone so that

$$
\|\nabla \theta(\cdot, t)\|_{0, \mu} \leq\left(\int_{0}^{t}\|\nabla u(\cdot, s)\|_{0, \mu} d s\right)\left\{\exp \left\{C \int_{0}^{t}\|\nabla u(\cdot, s)\|_{0, \mu} d s\right\}\right\}
$$

holds for each $t \leq T$ with $u=W[\delta, \phi]$ and $\theta=\Theta[\delta, \phi]$.

Proposition 2 states that the map $\Theta$ is bounded but not that it is continuous in the strong $C^{1, \mu}$ topology. The proof follows naturally from the idea to use the classical method of characteristics and ODE Gronwall type arguments. Similar ideas are needed below in the slightly more difficult proof of Proposition 3 and we will sketch them there; therefore we leave the details of the proof of Proposition 2 to the interested reader.

In order to proceed let us now take a fixed $\phi$, take a small number $\epsilon>0$ and associate to it the set

$$
\mathcal{I} \subset \mathcal{P}_{T}
$$

defined by

$$
\mathcal{I}=\left\{\delta(x, t) ; \delta(x, 0)=0,\|\nabla \delta(\cdot, t)\|_{0, \mu} \leq \epsilon, \forall t \leq T\right\} .
$$

Combining the bounds in the two previous propositions one can choose, for fixed $\phi$, a $T$ small enough so that

$$
\delta \mapsto \Theta[\delta, \phi]=\mathcal{S}[\delta]
$$

maps

$$
\mathcal{S}: \mathcal{I} \rightarrow \mathcal{I}
$$

Inspecting the bounds it is clear that it is sufficient to require

$$
T\|\nabla \times \phi\|_{0, \mu} \leq c \epsilon
$$

with an appropriate $c$ depending on $\mu$ alone. Leaving $\phi, \epsilon$ and $T$ fixed as above, the map $\mathcal{S}$ is Lipschitz in the weaker norm $C^{0, \mu}$ :

Proposition 3. There exists a constant $C$, depending on $\mu$ alone, such that, for every $\delta_{1}, \delta_{2} \in \mathcal{I}$, the Lipschitz bound

$$
\left\|\mathcal{S}\left[\delta_{1}\right]-\mathcal{S}\left[\delta_{1}\right]\right\|_{0, \mathcal{P}} \leq C\left\|\delta_{1}-\delta_{2}\right\|_{0, \mathcal{P}}
$$

holds.

It is essential that $\delta_{j} \in \mathcal{I}$, so that they are smooth and their gradients are small, but nevertheless this is a nontrivial statement. An inequality of the type

$$
\left\|\mathcal{S}\left[\delta_{1}\right]-\mathcal{S}\left[\delta_{1}\right]\right\|_{0, \mathcal{P}} \leq C\left\|\delta_{1}-\delta_{2}\right\|_{1, \mathcal{P}}
$$


is easier to obtain, but loses one derivative. This kind of loss of one derivative is a well-known difficulty in general compressible hyperbolic conservation laws. The situation is complicated in addition by the fact that the constitutive law $W$ depends on gradients. As we shall see, incompressibility saves one derivative. The heart of the matter is

Proposition 4. Let $\phi \in\left(C^{1, \mu}\right)^{3}$ be fixed. There exists a constant depending on $\mu$ alone so that

$$
\left\|W\left[\delta_{1}, \phi\right]-W\left[\delta_{2}, \phi\right]\right\|_{0 \mu} \leq C\left\|\delta_{1}-\delta_{2}\right\|_{0, \mu}\|\phi\|_{1, \mu}
$$

holds for any $\delta_{j} \in C^{1, \mu}$ with $\left\|\delta_{j}\right\|_{1, \mu} \leq 1$.

One could use the condition $\delta_{j} \in C^{1, \mu}$ with $\left\|\delta_{j}\right\|_{1, \mu} \leq M$ but then $C$ would depend on $M$ also.

Proof of Proposition 4. Denoting

$$
\begin{gathered}
u=W\left[\delta_{1}, \phi\right]-W\left[\delta_{2}, \phi\right], \\
\delta=\delta_{1}-\delta_{2}, \\
\psi(x)=\frac{1}{2}\left(\phi\left(x+\delta_{1}(x)\right)+\phi\left(x+\delta_{2}(x)\right)\right), \\
v(x)=\phi\left(x+\delta_{1}(x)\right)-\phi\left(x+\delta_{2}(x)\right), \\
\gamma=\frac{1}{2}\left(\delta_{1}+\delta_{2}\right)
\end{gathered}
$$

we write

$$
u=u_{1}+u_{2}
$$

with

$$
u_{1}=\mathbf{P}\left\{(\nabla \delta)^{*} \psi\right\}
$$

and

$$
u_{2}=\mathbf{P}\left\{(\mathbf{I}+\nabla \gamma)^{*} v\right\}
$$

Now the bound

$$
\left\|u_{2}\right\|_{0, \mu} \leq C\|\delta\|_{0, \mu}\|\phi\|_{1, \mu}
$$

is obtained in the same way as the bound in Proposition 1. (Actually $\phi$ Lipschitz is enough here.) The dangerous term is $u_{1}$ because it contains $\nabla \delta$. But here we can "integrate by parts" and write

$$
u_{1}=-\mathbf{P}\left\{(\nabla \psi)^{*} \delta\right\}
$$

because of incompressibility. The matrix $\nabla \psi$ is bounded in $C^{0, \mu}$ and the bound follows again easily, as the bounds in Proposition 1. This ends the proof of Proposition 4. We draw attention to the fact that the presence of the $*$ (transpose) operation is essential for "integration by parts" to be allowed. 
Returning to the proof of Proposition 3 we denote $\theta_{j}=\mathcal{S} \delta_{j}, u_{j}=W\left(\delta_{j}, \phi\right)$, $u=u_{1}-u_{2}, \theta=\theta_{1}-\theta_{2}$ and write

$$
\frac{\partial \theta}{\partial t}+\frac{u_{1}+u_{2}}{2} \cdot \nabla \theta+u \cdot \nabla\left(\frac{\theta_{1}+\theta_{2}}{2}\right)+u=0 .
$$

We consider the characteristics $X(a, t)$ defined by

$$
\frac{d X}{d t}=\frac{u_{1}+u_{2}}{2}(X, t), \quad X(a, 0)=a,
$$

and note that in view of Proposition 1 and the assumption $\delta_{j} \in \mathcal{I}$, the characteristics are well defined for $0 \leq t \leq T$, and their inverse $A(x, t)=X^{-1}(x, t)$ (the "back-tolabels" map) is defined too. Moreover,

$$
\sup _{t, a}\left|\frac{\partial X}{\partial a}\right| \leq C
$$

and

$$
\sup _{t, x}\left|\frac{\partial A}{\partial x}\right| \leq C
$$

hold with a constant $C$ depending on $\mu$ alone. Now consider the function

$$
F(x, t)=u \cdot \nabla\left(\frac{\theta_{1}+\theta_{2}}{2}\right)+u .
$$

Solving by the method of characteristics we obtain

$$
\theta(x, t)=-\int_{0}^{t} F(X(A(x, t), s), s) d s .
$$

Using Proposition 4 in conjunction with the bounds in Propositions 1 and 2 we see that $F(x, t)$ is bounded (uniformly in time) in $C^{0, \mu}$ :

$$
\sup _{t}\|F(\cdot, t)\|_{0, \mu} \leq C\|\phi\|_{1, \mu}\|\delta\|_{0, \mathcal{P}} .
$$

Compositions with the uniformly Lipschitz $X$ and $A$ are harmless and we obtain the desired result:

$$
\|\theta\|_{0, \mathcal{P}} \leq C\|\delta\|_{0, \mathcal{P}}
$$

This ends the proof of Proposition 3. The proof of Theorem 1 now follows by using successive approximations. Starting with a first guess $\delta_{1} \in \mathcal{I}$ we define inductively

$$
\delta_{n+1}=\mathcal{S} \delta_{n} \in \mathcal{I} .
$$

Proposition 3 can be used to show that the sequence $\delta_{n}$ converges rapidly in the $C^{0, \mu}$ topology to a limit $\delta$. Because $\mathcal{I}$ is convex it contains this weaker limit point, $\delta \in \mathcal{I}$. Because $\mathcal{S}$ has the weak Lipschitz property of Proposition 3 it follows that $\mathcal{S} \delta=\delta$. This actually means that $A=x+\delta(x, t)$ solves the active vector formulation of the Euler equations and that $u=W[\delta, \phi]$ solves the usual Eulerian formulation.

Now let us consider the case of decay at infinity. This case is instructive to look at because it illuminates the difference between $\phi, u, W$ on the one hand and $x, \delta, \Theta$ on the other hand; the function spaces need to be modified in a natural fashion to accommodate this difference. The issue of decay at infinity is both a physical one - the total kinetic energy must be defined, and a mathematical one - $\mathbf{P}$ must be defined. But apart from this, the decay at infinity requirement does not hinder the proof in any respect. 
Theorem 2. Let $\phi$ be a $C^{1, \mu}$ velocity that is square integrable,

$$
\int|\phi(x)|^{2} d x<\infty,
$$

and whose curl is integrable to some power $1<q<\infty$,

$$
\int|\nabla \times \phi(x)|^{q} d x<\infty .
$$

Then for $\epsilon$ sufficiently small there exist a time interval $[0, T]$ and a $C^{1, \mu}$ function $\delta(x, t)$ such that

$$
\sup _{t}\|\nabla \delta(\cdot, t)\|_{0, \mu} \leq \epsilon
$$

and such that $x+\delta(x, t)$ solves the active vector formulation of the Euler equation. The velocity corresponding to this solution belongs to $C^{1, \mu}$, is square integrable and the vorticity is integrable to power $q$.

The proof follows along the same lines as above. Because $\phi$ enters linearly in the expression for $W$ and because we control $\nabla \delta$ uniformly, issues of decay at infinity do not arise. In other words, the function space for velocities does not need to be a Banach algebra, rather a module over the Banach algebra of the $\delta$ variables, which need not decay at infinity.

\section{The Blow up issue}

Any solution of the Euler equation can be constructed using a sequence of near identity transformations. One starts out with

$$
\phi=u_{0}
$$

and solves for an interval of time $0 \leq t \leq t_{1}$ the active vector equation

$$
\begin{gathered}
\frac{\partial A}{\partial t}+u_{A} \cdot \nabla A=0, \\
u_{A}=\mathbf{P}\left((\nabla A)^{*} u_{0}(A)\right), \\
A(x, 0)=x .
\end{gathered}
$$

At time $t=t_{1}$ one resets

$$
\phi=u_{1}=u_{A}\left(\cdot, t_{1}\right)
$$

and solves the system above again for a new time interval $t_{1} \leq t \leq t_{2}$ and so one continues the solution. The local existence result guarantees that

$$
\left(t_{n+1}-t_{n}\right)\left\|\nabla u_{n}\right\|_{0, \mu} \geq c>0
$$

and during this time the solution $A(x, t)$ remains close to the identity in the sense that $\delta=A-x$ obeys

$$
\|\nabla \delta(\cdot, t)\|_{0, \mu} \leq \epsilon
$$

with a prescribed $\epsilon<<1$. Formula (32) then implies that

$$
\left\|\nabla u_{n}\right\|_{0, \mu} \leq K^{n}\left\|\nabla u_{0}\right\|_{0, \mu}
$$

with a fixed $K>1$. If the inequalities above would be sharp, then, of course, the time steps would have to decrease exponentially and the procedure would diverge in finite time. It is possible that for certain initial data the bounds may be 
overly pessimistic and the solution may exist for a long time. But with the present knowledge, if one desires long-lived solutions for arbitrary three dimensional data, then one needs to smooth either at the end of each step or during each time step. If one applies a smoothing procedure one evidently changes the problem and one introduces an artificial dissipation. There are many ways one could conceivable regularize the Euler equations. The physically correct energy dissipating equation is the Navier-Stokes equation. Unfortunately it is not known in three dimensions if the Navier-Stokes equations have globally defined unique solutions that converge to solutions of the Euler equations. Even in two dimensions, where the existence of smooth solutions is known for both the Euler and Navier-Stokes equations, the situation is not entirely trivial $([17],[18])$. The two dimensional situation is characterized by the absence of vortex stretching. In the case of the three dimensional Euler equations the vorticity magnitude evolves according to the stretching equation

$$
D_{t}(|\omega|)=\alpha|\omega|
$$

The stretching factor $\alpha$ is related to the vorticity magnitude through a principal value singular integral ([19]):

$$
\alpha(x, t)=P . V . \int D(\hat{y}, \xi(x, t), \xi(x+y, t))|\omega(x+y, t)| \frac{d y}{|y|^{3}} .
$$

Here $\hat{y}$ is the unit vector in the direction of $y, \xi(x, t)=\frac{\omega}{|\omega|}$ is the unit vector tangent to the vortex line passing through $x$ at time $t$ and $D$ is a certain geometric factor. The geometric factor is a smooth function of three unit vectors, has zero average on the unit sphere, $\int D d S(\hat{y})=0$ and vanishes pointwise when $\xi(x, t)= \pm \xi(x+y, t)$. Because $\alpha$ has the same order of magnitude as $|\omega|$, dimensional reasoning suggests blow up of the type one encounters in the ordinary differential equation $\frac{d m}{d t}=m^{2}$,

$$
\sup _{x}|\omega(x, t)| \sim \frac{1}{T-t} .
$$

But if the vorticity direction $\xi$ is smooth, then a geometric depletion of $\alpha$ occurs; this means that $\alpha$ is of the order of magnitude of velocity times the magnitude of the spatial gradient of $\xi$ (an inverse length scale, assumed to be finite). The two dimensional Euler equations correspond to the case $\xi=(0,0,1)$ and $\alpha=0$ identically. If

$$
\int_{0}^{T} \sup _{x}|\alpha(x, t)| d t<\infty
$$

then no blow up can occur. This idea of geometric depletion of nonlinearity has been investigated theoretically and numerically for the Euler equations and for a quasi-geostrophic active scalar equation $([19],[20],[21],[22],[23],[24])$. In the Eulerian-Lagrangian formulation of the Euler equation the role played by smooth stratifications can be explained in the following manner. Consider functions $w=w_{\psi}$ of the form

$$
w_{\psi}(x, t)=(\nabla A(x, t))^{*} \psi(A(x, t))
$$

associated to arbitrary vectors $\psi$. Alternately, one might consider solutions of

$$
D_{t}^{A} w+\left(\nabla u_{A}\right)^{*} w=0
$$


with initial data $\psi$. A particular example is provided by choosing $\psi=\phi$, i.e. $w_{\phi}=w_{A}$,

$$
w_{A}=(\nabla A)^{*} \phi(A)
$$

which obeys

$$
\nabla \times w_{A}=\nabla \times u_{A}=\omega_{A} .
$$

Because the vorticity

$$
\omega_{A}=\nabla \times u_{A}
$$

satisfies

$$
\omega_{A}=(\nabla A)^{-1} \zeta(A)
$$

it follows that

$$
\omega_{A}(x, t) \cdot w_{\psi}(x, t)=\zeta(A(x, t)) \cdot \psi(A(x, t))
$$

holds for any $\psi$ or, in other words,

$$
D_{t}\left(\omega_{A} \cdot w\right)=0
$$

holds for any solution $w(x, t)$ of (40). Global regularity of a solution of the Euler equations would follow from (45) if one could find a sufficient family of vectors $\psi$. By a sufficient family for the initial velocity $\phi$ and the time interval $[0, T]$ we mean a family of vectors $\psi$ such that there exists a nonnegative function $\gamma(t)$ with $\int_{0}^{T} \gamma d t<\infty$ such that

$$
\left|\omega_{A}(x, t)\right| \leq \gamma(t) \sup _{\psi}\left|\omega_{A}(x, t) \cdot w(x, t)\right|
$$

holds for $0 \leq t \leq T$. A sufficient family for all two dimensional flows is provided by just one $\psi, \psi=(0,0,1)$, with $\gamma=1$. Generalizations would consist of situations in which one could find sufficient families that depend on the initial data and time and take locally the role played in $2 \mathrm{D}$ by the vertical direction.

The blow up issue becomes, in terms of $A$, a question of formation of infinite gradients in conserved quantities. This is similar to the case of hyperbolic conservation laws but with the significant difference that the underlying characteristic flow is volume-preserving: $\operatorname{det}(\nabla A)=1$, the matrix $\nabla A$ is invertible and

$$
\left((\nabla A(x, t))^{-1}\right)_{i j}=\frac{1}{2} \epsilon_{i m n} \operatorname{Det}\left[e_{j} ; \frac{\partial A}{\partial x_{m}} ; \frac{\partial A}{\partial x_{n}}\right]
$$

holds, where $e_{j}=\left(\delta_{j k}\right)$ is the canonical basis in $\mathbf{R}^{3}$. Consider the Euler-Lagrange label differentiation

$$
L_{j}^{A}=\frac{1}{2}\left(\epsilon_{i m n} \epsilon_{j k l} \frac{\partial A_{k}}{\partial x_{m}} \frac{\partial A_{l}}{\partial x_{n}}\right) \frac{\partial}{\partial x_{i}} .
$$

From the commutation relation (20) and the $A$ equation (23) it follows that

$$
\left[D_{t}^{A}, L_{j}^{A}\right]=0
$$

holds for any $j=1,2,3$. This commutation relation simply says that in Lagrangian coordinates, time and label derivatives commute. Note from formulas (44) and (47) that

$$
\frac{1}{2} \epsilon_{p i l} \operatorname{Det}\left[\zeta(A) ; \frac{\partial A}{\partial x_{i}} ; \frac{\partial A}{\partial x_{l}}\right]=\omega_{A}^{p}
$$


It is clear now that $D_{t}^{A}$ commutes with $\Omega_{A}=\omega_{A} \cdot \nabla$ because it is represented in terms of $L_{j}^{A}$ :

$$
\Omega_{A}=\zeta_{j}(A) L_{j}^{A} .
$$

Observe that, in view of the definition of the operators $L_{j}^{A}$,

$$
L_{j}^{A}=\left((\nabla A(x, t))^{-1}\right)_{k j} \frac{\partial}{\partial x_{k}},
$$

it follows that

$$
\left((\nabla A(x, t))^{-1}\right)_{i j}=L_{j}^{A}\left[x_{i}\right]
$$

on the other hand

$$
D_{t}^{A}\left(x_{i}\right)=u_{A}^{i}
$$

holds, so from the commutation relation (49) we obtain

$$
D_{t}^{A}\left((\nabla A(x, t))^{-1}\right)_{i j}=L_{j}^{A}\left(u_{A}^{i}\right)
$$

This equation, which could have been derived also directly from (30), implies the vorticity equation because of (29):

$$
D_{t}^{A} \omega_{A}=\zeta_{j}(A) L_{j}^{A}\left(u_{A}\right)=\Omega_{A}\left(u_{A}\right) .
$$

Because of the result in [2] and (29), it is clear that the finiteness of

$$
\int_{0}^{T}\left\|(\nabla A(\cdot, t))^{-1}\right\|_{L^{\infty}(d x)} d t
$$

implies regularity. Or, using (47), we deduce that the finiteness of

$$
\int_{0}^{T}\|\nabla A(\cdot, t)\|_{L^{\infty}(d x)}^{2} d t
$$

implies regularity. Let us now introduce the matrix

$$
C_{i j}^{A}(x, t ; z)=(\nabla A(x+z, t))_{i m}\left((\nabla A(x, t))^{-1}\right)_{m j},
$$

and call it the Euler-Lagrange calibrator. The formula

$$
C_{i j}^{A}(x, t ; z)=L_{j}^{A(x, t)}\left(A_{i}(x+z, t)\right)
$$

shows that the calibrator measures the response of the Eulerian translation to an infinitesimal Lagrangian translation. Note that

$$
C_{i j}^{A}(x, t ; 0)=\delta_{i j} .
$$

The calibrator is a quotient of gradients at different locations and therefore locally spatially uniform, temporally arbitrary changes like dilations do not affect it. The vorticity equation (56) can be expressed in terms of the Euler-Lagrange calibrator (25]):

$$
D_{t}^{A} \omega_{A}^{i}=\left\{\frac{1}{4 \pi} P . V . \int D\left(\zeta, C^{A} \zeta, C_{\cdot, p}^{A}\right) \sigma_{i l}(\hat{z}) \frac{d z}{|z|^{3}}\right\} \frac{\partial A_{p}(x, t)}{\partial x_{l}} .
$$

Note that

$$
\omega_{A}^{i} \frac{\partial A_{p}(x, t)}{\partial x_{i}}=\zeta_{p}(A(x, t))
$$


is bounded. It is therefore natural to conjecture that the smoothness of $C^{A}$ prevents finite time blow up for the Euler equations. This conjecture is true for the quasigeostrophic active scalar. The interested reader is referred to ([25]) for details.

The blow up question for the Euler equations remains open. Numerical calculations provide insight and hints, but the answer will have to be analytical. The considerations above point towards a possible incompressible dispersive effect that hinders blow up: as the gradients of $A$ become large the resulting rapid (15) and nonuniform 55) motion disperses the large gradients. This might cause instability of blow up or perhaps its suppression.

\section{ACKNowledGments}

The author thank Diego Cordoba, Charles Fefferman and Julian Hunt for helpful comments. This research was supported in part by NSF- DMS9802611. Partial support of AIM and the hospitality of the Princeton Mathematics Department are gratefully acknowledged.

\section{REFERENCES}

[1] A. Majda, Vorticity and the mathematical theory of incompressible flow, Comm. Pure Appl. Math. S39 (1986), 187-220. MR 87j:76041

[2] J.T. Beale, T. Kato, A. Majda, Remarks on the breakdown of smooth solutions for the 3-D Euler equations, Comm. Math. Phys. 94 (1984), 61-66. MR 85j:35154

[3] H. K. Moffatt, The degree of knotedness of tangled vortex lines, J. Fluid Mech. 35 (1969), 117-129.

[4] V. I. Arnold, B. A. Khesin, Topological methods in hydrodynamics, Ann. Rev. Fl. Mech. 24 (1992), 145-166. MR 93d:58020

[5] A. J. Chorin, Vorticity and Turbulence, Applied Mathematical Sciences 103, (1994), SpringerVerlag, New York. MR 95m:76043

[6] U. Frisch, Turbulence, Cambridge University Press, (1995), Cambridge. MR 98e:76002

[7] J. Serrin, Mathematical principles of classical fluid mechanics (S. Flugge, C. Truesdell, Eds.), Handbuch der Physik, 8 (1959), 125-263, p.169. MR 21:6836b

[8] M. E. Goldstein, Unsteady vortical and entropic distortion of potential flows round arbitrary obstacles, J. Fluid Mech. 89 (1978), 433-468.

[9] M. E. Goldstein, P. A. Durbin, The effect of finite turbulence spatial scale on the amplification of turbulence by a contracting stream, J. Fluid Mech. 98 (1980), 473-508.

[10] J. C. R. Hunt, Vorticity and vortex dynamics in complex turbulent flows, Transactions of CSME 11 (1987), 21-35.

[11] G. A. Kuzmin, Ideal incompressible hydrodynamics in terms of the vortex momentum density, Phys. Lett. A 96 (1983), 88-90.

[12] V. I. Oseledets, On a new way of writing the Navier-Stokes equation. The Hamiltonian formalism, Commun. Moscow Math. Soc. (1988), Russ. Math. Surveys 44 (1989), 210-211. MR 91e:58173

[13] T. F. Buttke, Velicity methods: Lagrangian numerical methods which preserve the Hamiltonian structure of incompressible fluid flow, in Vortex flows and related numerical methods, NATO Adv. Sci. Inst. Ser. C Math. Phys. Sci., J.T. Beale et al., Eds. 395 (1993), Kluwer (Dordrecht), 39-57. MR 94m:76093

[14] J. H. Maddocks, R. L. Pego, An unconstrained Hamiltonian formulation for incompressible fluid flow, Comm. Math. Phys. 170 (1995), 207-217. MR 96a:76085

[15] D. Ebin, J. Marsden, Groups of diffeomorphisms and the motion of an incompressible fluid, Ann. of Math. 92 (1970), 102-163. MR 42:6865

[16] T. Kato, Non-stationary flows of viscous and ideal fluids in $\mathbf{R}^{3}$, J. Funct. Anal. 9 (1972), 296-309. MR 58:1753

[17] A. Bertozzi, P. Constantin, Global regularity for vortex patches, Comm. Math. Phys. 152 (1993), 19-28. MR 94b:35221

[18] P. Constantin, J. Wu, The inviscid limit for non-smooth vorticity, Indiana Univ. Math J. 45 (1996), 67-81. MR 97g:35129 
[19] P. Constantin, Geometric and analytic studies in turbulences, in Trends and Perspectives in Appl. Math., L. Sirovich, ed., Appl. Math. Sciences 100, Springer-Verlag, (1994). MR 95f:76017

[20] P. Constantin, A. Majda, E. Tabak, Formation of strong fronts in the 2D quasi-geostrophic thermal active scalar, Nonlinearity 7 (1994), 1495-1533. MR 95i:76107

[21] P. Constantin, C. Fefferman, A. Majda, Geometric constraints on potentially singular solutions for the 3-D Euler equations, Commun. in PDE 21 (1996), 559-571. MR 97c:35154

[22] K. Ohkitani, M. Yamada, Inviscid and inviscid-limit behavior of a surface quasi-geostrophic flow, Phys. Fluids 9 (1997), 876 -882. MR 97m:76032

[23] D. Cordoba, On the geometry of solutions of the quasi-geostrophic active scalar and Euler equations, Proc. Nat. Acad. Sci. USA 94 (1997), 12769-12770.

[24] P. Constantin, Q. Nie, N. Schoerghofer, Nonsingular surface quasi-geostrophic flow, Phys. Lett. A 241 (27 April 1998), 168-172. MR 99a:76031

[25] P. Constantin, An Eulerian-Lagrangian approach to incompressible fluids, http://www. aimath.org/preprints/99/constantin.dvi

Department of Mathematics, The University of Chicago, Chicago, Illinois 60637-1546

E-mail address: const@cs.uchicago.edu 